\title{
Assessing the Impacts of Open Hypermedia Problems on Structural Computing
}

\author{
Nikos Karousos $^{1,2}$ and Nikos Tsirakis ${ }^{1,2}$ \\ ${ }^{1}$ Research Academic Computer Technology Institute, \\ 15600, Rion, Greece \\ karousos@cti.gr \\ ${ }^{2}$ Department of Computer Engineering \& Informatics, \\ University of Patras, 26500 Rion Greece \\ tsirakis@ceid.upatras.gr
}

\begin{abstract}
This paper is focusing on some interesting issues about service publicity and usability while trying to move from the classic Open Hypermedia Systems (OHSs) to structural aware environments. Although the step towards structural computing provides a stable basis for new generation systems, these systems inherit from OHSs some long-period problems and aspects. Moreover, new usability problems are rising due to the increment of structural servers' complexity.
\end{abstract}

\section{Introduction}

In today's computer science community, the dominant trend is towards open systems. Open hypermedia systems (OHSs) employed a variety of techniques in order to spread the hypermedia services usability. These systems have tried to add new hypermedia functionality to the world.

Although open hypermedia systems are characterized by structures and openness they were usually supporting only navigational approach [5] and were lacking of supporting towards multiple domains with different structural abstractions. Research led to structural computing [7] in order to solve some data organization problems. Structural computing in a way generalizes the techniques used by open hypermedia [1]. The structural computing idea was the result of the philosophy: "primacy of structure over data" [10]. In general it contributes to a generalization of the hypermedia field.

Currently some interesting efforts of moving from OHS to structural aware systems have been reported [6, 11]. This step has shifted current OHSs drawbacks from OHS community to the structural computing area. These issues are critical while aiming to apply structural principles to real world application systems, due to their involvement in the structural services publicity and usability. Namely, the low publicity of OHSs, the unsatisfactory level of hypermedia service provision and the increment of structure service complexity are influencing the adoption of the structural computing theory into hypermedia systems. 
This paper presents the critical issues discussed above and suggests some actions aiming to a stable progress of structural computing research.

\section{From Open Hypermedia Systems to Structural Aware Environments}

The step from open hypermedia theory to structural computing tried to unify hypermedia domains under a common conceptual foundation. Furthermore, the notion of the structure awareness was the key point for this approach. Although structural computing research is in primary level, it still has some notable advantages [10]:

- Interoperability: Different applications can share their objects and their associations under different structural abstractions.

- Efficiency: Some intelligent operations that systems can support.

- Multiple domains: Provide co-existence of different domains under the same framework.

- Complexity: As SC has an object-oriented philosophy of computing, some of the previous problems are now easy-detected and addressed. Since the operations are implemented in an object oriented way, the complexity of the structural environments architecture is reduced.

Undoubtedly these advantages signify a promising future for structural computing but unfortunately there are also some remarkable problems that arise. Most of them derive both from the existed OHSs' problems and the structural aware environments' characteristics. An interesting user-based study about the usability of OHSs [2] reinforces some of the following issues.

\subsection{Publicity}

Open Hypermedia Systems have not yet been well-known to public and don't have a global usage [8]. There are three underlying categories that agglomerate the reasons for this:

Research. The Open Hypermedia Researches act in a closed research environment. Apart from the navigational point of view, other hypermedia fields (ubiquitous, taxonomic, etc) that have already been analyzed, have not yet reached a satisfactory level of publicity. Furthermore, it is difficult for new researches to contribute in the hypermedia research area due to the limited information resources.

Systems' Promotions. Open Hypermedia Systems are very powerful tools, but their benefits are not promoted enough to the public. Consequently, in the users and developers' area, the OHSs' products have not been presented successfully. A developer who wants to add a spatial or a taxonomic representation into his application does not know where to look for it and how he can use an available hypermedia service. In most cases he tries to implement custom solutions. 
Web Publicity. It seems that (like some current working groups and organizations) a centralized (web based) information point, which can provide the appropriated fundamental information to the public, is still missing. Like other existing groups (World Wide Web Consortium, Internet Engineering Task Force, etc), the Open Hypermedia Working Group should try to continue (or revise) its work and publish an up-to-date web site that can inform a visitor about the Open Hypermedia Research.

\subsection{Service Provision}

Hypermedia services were always trying to reach an open set of potential users in order to increase the enrichment of the hypermedia functionality to the software systems. These efforts were not completed appropriately due to absences that derived from systems architecture, developer supporting, discovering problems and providing policies. These architectures lack of:

- developer support framework. [3]

- standardized methodologies and protocols for service provision. [4]

- add-hoc service usage implementations and web integration efforts [4].

- service discovery system [3].

- flexible pricing policy [9].

- service oriented hypermedia systems that can provide partial services instead of the complete hypermedia system [9].

\subsection{Complexity Consequences}

The architecture of hypermedia services was always complicated. The shift from data to structure problematizes models of representation [8] and results more complex structural services. Keeping the same service's API while both the architecture and the service kernel are changing is not an easy task. Consequently, although structural aware environments support multiple domains and make their parallel usage feasible, the effort for the developer to use only one service seems larger in structural environments than in classic hypermedia systems.

On the other hand, the interoperability issues among new structural environments are not fully faced yet.

\section{Assuring a Successful Step}

There is a general need for some conceptual decisions towards the difficulties that have been mentioned in the above sections. Specifically we can highlight them in the following three fields.

Publicity. It's a common belief that research community looks for a way to improve the promotion of their results in order to broaden their knowledge and ideas around this computer science field. As a first step to this direction, a web-based information place should be created, in order for researchers to be informed about structural computing and structural aware environments. 
Service provision. The absence of both developer's support frameworks and the standardization of service provision techniques will raise many difficulties in development procedure, when incorporating structural computing infrastructure. There is also a necessity to implement a service discovery system and to become an inherent part of any system with structural computing philosophy. This will contribute to the widespread of the service provision. Finally, the adoption of service oriented architectures can boost the usage of structural services from users and developers.

Complexity. Since the research community is still in the progress of establishing an agenda for building structural computing environments, we ought to work on keeping clear structural service APIs that are easy to use. Furthermore, we should try to hide complicated structural operations in the internal of the services.

\section{Conclusions}

Structural computing is still in its early stages of research and is characterized as a revolutionary new computing paradigm. The step from the structural computing principles to the structural aware environments and applications is a very difficult task. In order this effort to avoid closed theoretical approaches or implementations of non wide acceptance systems, the drawbacks of open hypermedia systems and the structural service provision issues have to be faced. The structural computing community should take into consideration the findings of the OHSs history towards the efficient creation of the new generation systems.

\section{References}

1. Anderson, K. M., Sherba, S. A., Lepthien, W. V. (2003). Structure and behavior awareness in themis. In Proceedings of the ACM Hypertext 2003 Conference, pp.138-147, (Nottingham, England).

2. Hicks, David L. (2002). In search of a user base: Where are the B's? Proceedings of MetaInformatics 2002, Esbjerg, Denmark, August 8-10, 2002.

3. Karousos, N., Pandis, I. (2003). Developer Support in Open Hypermedia Systems: Towards a Hypermedia Service Discovery Mechanism. Proceeding of Metainformatics Symposium (MIS' 03), (Graz, Austria).

4. Karousos, N., Pandis, I., Siegfried, R., and Tzagarakis, M. (2003). Offering Open Hypermedia Services to the WWW: A Step-by-Step Approach for the Developers. In Twelfth International World Wide Web Conference WWW2003, (Budapest, Hungary), pp. 482-489.

5. Michail Vaitis, Manolis Tzagarakis, George Gkotsis (2004). An Engineering Perspective on Structural Computing-Developing Component-Based Open Hypermedia Systems. International Workshop on Web Engineering, ACM Hypertext 2004.

6. Millard, D. (2003). Discussions at the Data Border: From Generalised Hypertext to Structural Computing. Special Issue on Structural Computing, Journal of Network and Computer Applications, 26, 1, (January):pp. 95-114. 
7. Peter. J. Nürnberg, J. J. Leggett, E. R. Schneider (1997). As we should have thought, in: Proceedings of the 1997 ACM Hypertext Conference, ACM, ACM Press, Southampton, UK, 1997, pp. 96-101.

8. Peter. J. Nürnberg, Schraefel, M. C. (2003). Relationships Among Structural Computing and Other Fields. Special Issue on Structural Computing, Journal of Network and Computer Applications, 26, 1, (January).

9. Shackelford, D. E., Smith J. B., Smith F. D. (1993). The Architecture and Implementation of a Distributed Hypermedia Storage System. In Proceedings of the 1993 ACM Hypertext Conference, (Seattle, WA, Nov), ACM press, pp. 1-13.

10. Uffe K. Wiil, Peter J. Nürnberg, David L. Hicks, (2003). Structural Computing - Research Directions Systems and Issues. Special Issue on Structural Computing, Journal of Network and Computer Applications, 26, 1, (January), 3-9.

11. Weigang Wang (2003). Co-existence and visualization of multi-domain hypertext structures. Special Issue on Structural Computing, Journal of Network and Computer Applications, 26, 1, (January). 